\title{
T1 inversion recovery pulse sequence: could it replace T1 spin echo sequence in neuroimaging by improving tissue contrast?
}

\author{
Mohamed Shawky, Rehab Habib and Ahmed Elsawaf*
}

\begin{abstract}
Background: $T 1$ inversion recovery (T1IR) sequence improved tissue contrast by providing higher gray matterwhite matter contrast ratio (GM-WM contrast ratio) and higher lesion contrast noise ratio (CNR). This study aims to highlight its significance in the evaluation of space-occupying lesions whether intra-axial or extra-axial and also in multiple sclerosis (MS) by comparing it with T1 spin echo (T1SE) sequence.

Result: In a total of 50 patients, 14 patients with extra-axial lesions, 18 patients with intra-axial lesions, and 18 patients with multiple sclerosis were included. The CNR was significantly higher for pre-contrast T1IR images than for pre-contrast T1SE (- 13.04 (1.20) vs -7.73 (0.70); $p$ value < 0.01). After giving intravenous contrast media, CNR in T1SE was higher than T1IR (11.14 (1.75) vs $9.41(1.83)$ ) without statistical significance ( $p$ value $=0.19)$ and CNR was higher in T1IR than T1SE in lesions with low enhancement ratio (ER). As well, the overall number of lesions was higher on T1IR especially in MS (10.67 (2.26) vs 3.89 (1.05); $p$ value $<0.01)$

Conclusion: On pre-contrast sequences, T1IR could be used as an added sequence in most brain lesions giving higher lesion CNR. After giving intravenous contrast media, T1IR could be used in lesions with low ER. It also could be used in situations in which gadolinium injection is contraindicated and also could be used in follow-up of MS patient by detecting a higher number of lesions that can be easily missed in T1SE.
\end{abstract}

Keywords: $\mathrm{T1}$ inversion recovery, $\mathrm{T1}$ spine echo, Neuroimaging, Tissue contrast

\section{Background}

Inversion recovery sequences are commonly used as FLAIR (fluid attenuated inversion recovery) and STIR (short tau inversion recovery) by suppressing MR signals from CSF and fat, respectively. However, it could also be used to generate T1-weighted images that are called T1 inversion recovery (T1IR) [1].

Through reducing the acquisition time in this sequence and increasing its spatial resolution by applying multishot echo planar imaging and fast spin echo imaging, it became practical to be used in neuroimaging [2]. Furthermore, it improved tissue contrast by providing higher gray matter-white matter (GM-WM) contrast

\footnotetext{
* Correspondence: Ahmedage51@yahoo.com

Department of Radiodiagnosis, Faculty of Medicine, Menoufia University, Menoufia 32511, Egypt
}

ratio and higher lesion contrast noise ratio (CNR), so it could be used in the evaluation of neoplastic space-occupying lesion whether extra-axial or intra-axial [3].

It also could be used in the diagnosis, monitoring disease progression, and treatment efficacy in multiple sclerosis (MS) as it detects the black hole lesions that show better correlation with clinical disability [4]. Conversely, the correlation between the current magnetic resonance (MR) measures and clinical disability measures is still weak (that is called MRI/clinical dissociation), making the current MRI sequences inadequate in the monitoring of disease activity and insufficient in the assessment of treatment efficacy [5-7]. T1IR, by providing higher GM-WM contrast ratio and lesion CNR, might aid in revealing a higher number of cortical lesions in MS that go undetected in conventional MR 


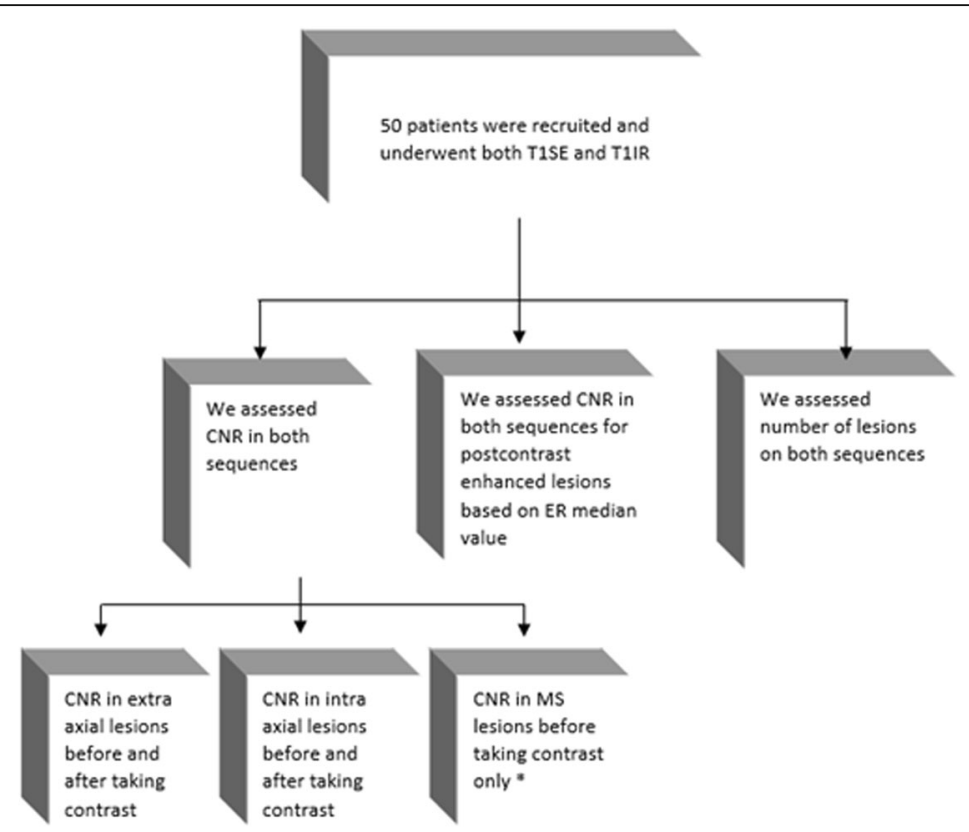

*For MS, 17 from 18 patients did not have active lesions so their SI did not change on post contrast study. Therefore, we didn't assess the post contrast values in them

Fig. 1 Flow chart of data analysis

techniques, making it more promising for accurate quantification of lesion load and disease burden [8-10].

The goal of this study is to highlight the significance of T1IR sequence in neuroimaging in the evaluation of neoplastic space-occupying lesions whether intra-axial or extra-axial and also in the evaluation of demyelinating diseases such as multiple sclerosis, by comparing it to the conventional T1 spin echo (T1SE) to determine which of them would be the most valuable sequence.

\section{Methods and materials}

This study was carried at Menoufia University Hospitals, and all study procedures were approved by the ethical committee of Menoufia Faculty of Medicine and after taking written informed consent from patients involved in the study; it was prospectively performed from August 2017 to July 2018. Fifty patients were recruited and subjected to MRI imaging. We included neoplastic space-occupying lesions whether primary or metastatic. Other nonneoplastic space-occupying lesions were excluded (infective or inflammatory). We also included demyelinating lesions due to multiple sclerosis and excluded demyelinating lesions due to viral, metabolic, toxic, and vascular causes. All MR examinations were performed using a 1.5-T MR scanner (Toshiba Excelart Vantage, Japan) and standard 16-channel headcoil. We obtained T1SE pre-contrast and postcontrast (axial), T1IR pre-contrast and post-contrast (axial), T2WI (axial), FLAIR (axial), T2WI (coronal), T1WI (sagittal), and diffusion-weighted (axial) images. Post-contrast images were performed $4 \mathrm{~min}$ after the injection of a gadolinium contrast agent (gadopentetic acid, Gd DTPA, $0.1 \mathrm{mmol} / \mathrm{kg}$, Magnevist, Bayer Health Care, German) by manual injection followed by $20 \mathrm{ml}$ saline flush. Scan parameters for T1SE (axial) were as follows: repetition time (TR)/echotime (TE) $465 \mathrm{~ms} / 12 \mathrm{~ms}$, bandwidth $140 \mathrm{kHz}$, slice thickness $5 \mathrm{~mm}$, matrix $208 \times 352$, flip angle 90, number of excitations (NEX) 1, field of vision (FOV) $24 \mathrm{~cm}$, number of slices 20 , and scan time $2 \mathrm{~min} 45 \mathrm{~s}$. Scan parameters for T1IR (axial) were as follows: repetition time (TR)/echotime (TE) $3550 \mathrm{~ms} / 18 \mathrm{~ms}$, bandwidth $98 \mathrm{kHz}$, slice thickness 5 mm, matrix $192 \times 336$, flip angle 90:180, number of excitations (NEX)1, field of vision (FOV) $24 \mathrm{~cm}$, number of slices 20 , and scan time 2 min $58 \mathrm{~s}$.

In our study, images were interpreted by two radiologists-blinded to clinical data-with experience over 10 years, in conjoint reading sessions, and then, the final decision was taken by discussion and consensus. These images were analyzed in both T1SE (axial) and T1IR (axial) sequences with respect to lesion number and signal intensity (SI). The SI was calculated digitally by drawing a circular region of interest (ROI) on the lesion, approximately $100 \mathrm{~mm}^{3}$. The images of interest were displayed side by side on the workstation (Aze Virtual Place FujinRaijin 310). 


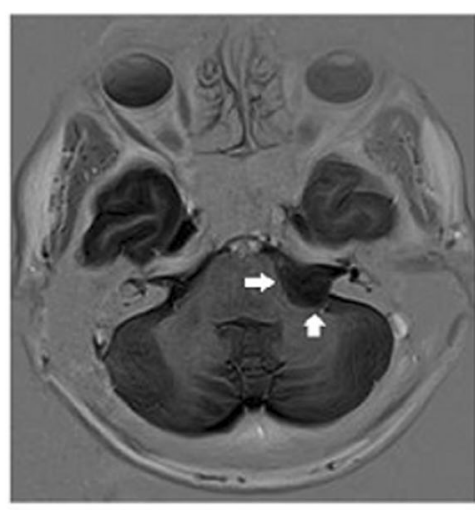

(a) TIIR pre-contrast

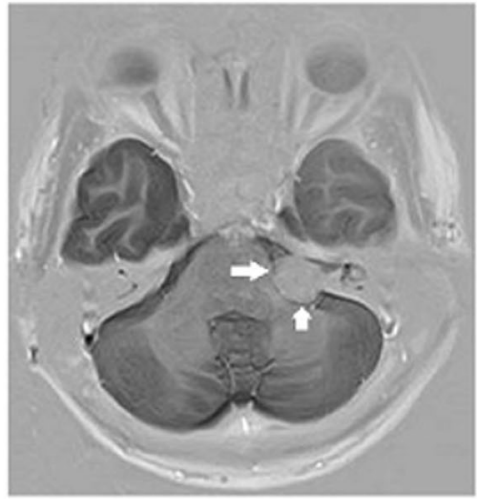

(c) TIIR post contrast

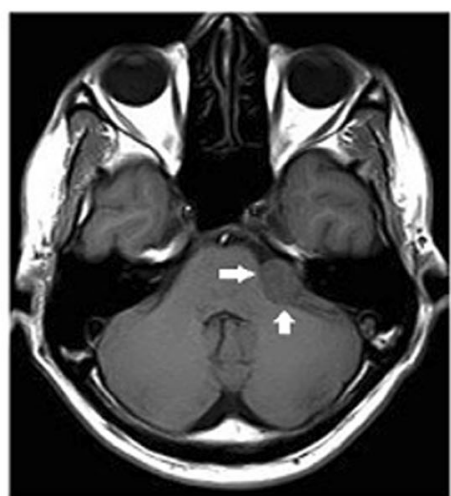

(b) TISE pre-contrast

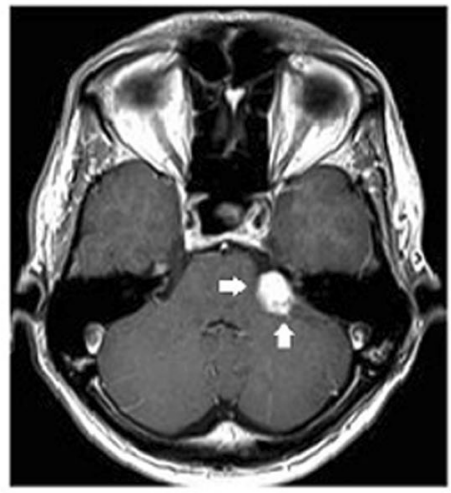

(d) T1SE post contrast

Fig. 2 Accidentally discovered posterior fossa mass. a T1IR pre-contrast. b T1SE pre-contrast. Posterior fossa lesion at left cerebellopontine angle that was better identified in T1IR than in T1SE as it appears with hypointense signals and higher lesion CNR than surroundings in T1IR with better delineation between its border and the adjacent left middle cerebellar peduncle (arrows in a, arrows in b). c T1IR post-contrast. d T1SE postcontrast. After giving intravenous contrast media, the lesion appeared with homogenous enhancement in both sequences with no superiority of each of these sequences (arrows in $\mathbf{c}$, arrows in $\mathbf{d}$ )

\section{Statistical analysis}

First, we compared the degree of lesion contrast noise ratio (CNR) between T1SE and T1IR before and after giving intravenous contrast media. CNR was defined as the difference between the lesion signal intensity and white matter signal intensity (SI) divided by the lesion signal intensity standard deviation (SD). We further compared the degree of CNR between T1SE and T1IR sequences according to each type of lesion into three subgroups (extra-axial, intra-axial, MS).

After giving intravenous contrast media, we compared the CNR between T1SE and T1IR sequences according to enhancement ratio (ER) into two subgroups (low enhancement ratio and high enhancement ratio): $\mathrm{ER}=$ post-contrast (lesion contrast ratio)/pre-contrast (lesion contrast ratio). Lesion contrast ratio was defined as SI of lesion divided by SI of white matter. The median overall value of all lesion ER was (0.68), so we divided lesions into high enhancement ratio and low enhancement ratio groups based on this value. Finally, we assessed the difference between T1SE and T1IR sequences after giving intravenous contrast media according to number of enhanced lesions (Fig. 1). All statistical analysis was performed on Microsoft Excel 2016 using a one-tailed

Table 1 Difference between T1IR and T1SE in CNR and number of lesions

\begin{tabular}{llll}
\hline MR sequences & Pre-contrast CNR & Post-contrast CNR & Number of lesions \\
\hline T1IR & $-13.04(1.20)^{*}$ & $9.41(1.83)$ & $4.94(0.92)^{*}$ \\
T1SE & $-7.73(0.70)$ & $11.14(1.75)$ & $2.36(0.37)$ \\
$p$ value & $<0.01$ & 0.19 & $<.01$ \\
\hline
\end{tabular}




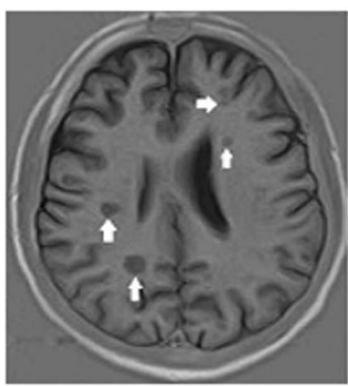

(a) $T 11 R$

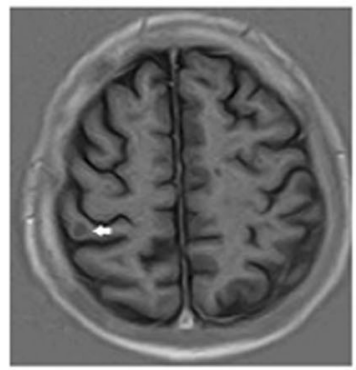

(c) $T I I R$

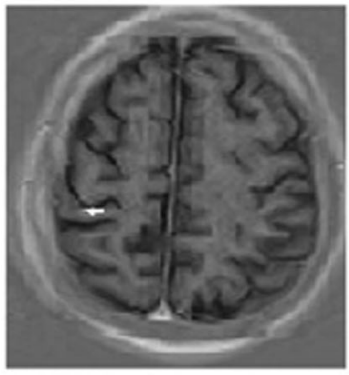

(e) TlIR post contrast

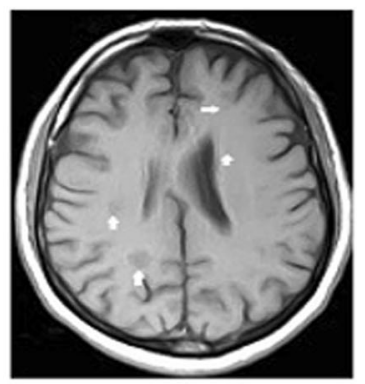

(b) T1SE

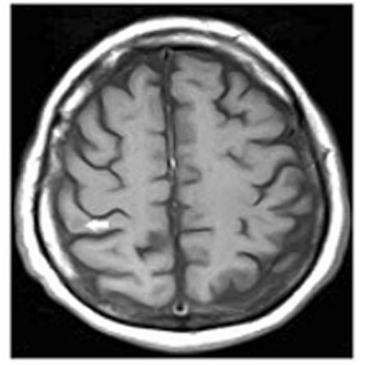

(d) TISE

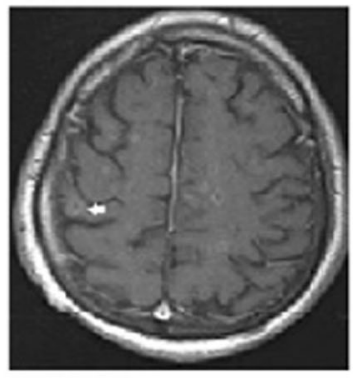

(f) TISE postcontrast

Fig. 3 Male patient, 50 years old, diagnosed as metastatic bronchogenic carcinoma to the brain. a T1IR. b T1SE. It revealed that T1IR aided in better delineation of lesions and also in detection of lesions (arrows in a) that were not clearly seen in T1SE (arrows in $\mathbf{b}$ ). c T1IR. d T1SE. It revealed that T1IR showed a right cortical lesion (arrow in $\mathbf{c}$ ) that was barely detected in T1SE (arrow in $\mathbf{d}$ ). e T1IR postcontrast. f T1SE post-contrast. The right cortical lesion enhancement could not be assessed accurately in T1IR post-contrast image due to motion artifacts (arrow in e), and there was also a faint ring enhancement in this lesion in T1SE post-contrast image (arrow in $\mathbf{f}$ )

paired $t$ test. A $p$ value of less than 0.05 was considered statistically significant.

\section{Results}

During the study period, a total of 50 patients were included (21 males and 29 females). The mean age was 43 years old (age range 19-73 years). Extra-axial lesions were identified in 14 patients (5 with meningioma, 3 with metastasis of unknown origin, 2 with vestibular shwannoma, 2 with metastasis from breast carcinoma, 1

with metastasis from rhabdomyosarcoma, and 1 with secondary lymphoma); meanwhile, intra-axial lesions were identified in 18 patients (5 with metastasis from bronchogenic carcinoma, 5 with glioblastoma, 4 with anaplastic astrocytoma, 2 with metastasis from breast carcinoma, and 2 with metastasis from unknown origin) and MS lesions were also identified in 18 patients. The SI for both the lesion and white matter was recorded then CNR was calculated for both pre-contrast and post-contrast study.

\section{Comparing T1SE and T1IR based on CNR}

The CNR was significantly higher in pre-contrast T1IR than in pre-contrast T1SE with mean value - 13.04 (1.20) vs $-7.73(0.70)$ and with $p$ value $<0.01$ (Fig. 2). Furthermore, the CNR was higher in post-contrast T1SE than in post-contrast T1IR, although difference did not achieve statistical significance with mean value 11.14 (1.75) vs 9.41 (1.83) and $p$ value $=0.19$ as shown in Table 1 .

\section{Subgroup analysis \\ Comparing T1SE and T1IR based on type of lesion}

The CNR was significantly higher in T1IR before contrast injection at all types of lesions either extra-axial, intra-axial, or MS with mean values - 13.90 (2.86), 12.87 (2.40), and -12.69 (1.63) and with $p$ values 0.02 , < 0.05 , and 0.01 , respectively (Figs. 2,3 , and 4 ). On the other side, post-contrast T1SE achieved higher efficacy without statistical significance for extra-axial lesions with mean value 18.48 (3.48) vs 13.17 (3.01) and with $p$ value 0.06 . For intra-axial lesions, post-contrast T1SE lesion CNR was, as well, higher without statistical significance with mean value 17. 08 (2.65) vs 16.46 (4.07) and with $p$ value 0.45 . For MS, 17 from 18 patients did not have active lesions, so their SI did not change on post-contrast study. Therefore, we did not assess the post-contrast values in them (Table 2).

\section{Comparing T1SE and T1IR based on ER}

The mean CNR value was significantly higher in T1IR in the weak enhancement ratio group with mean value 18.91 (2.68) vs 1.05 (0.59). Meanwhile, it was significantly higher in T1SE in the high enhancement group (21.24 (1.92) vs $0.64(0.37))$ and the results were statistically significant in both groups ( $p$ value $<0.01)$ as shown in (Table 3 ).

\section{Comparing T1SE and T1-IR based on number of lesions}

The overall number of lesions was significantly higher in T1IR than in T1SE with mean value $4.94(0.92)$ vs 2.36 (0.37) and $p$ value $<0.01$ (Table 1 ). Regarding subgroup analysis, we found that T1IR was statistically significant in MS group as regards to the number of lesions (10.67 (2.26) vs 3.89 (1.05); $p$ value $<0.01$; Fig. 4), but it showed no difference in extra- or intra-axial lesions with mean 


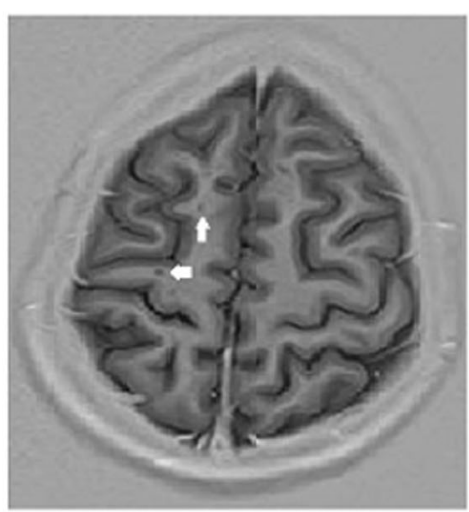

(a) $T 1 I R$

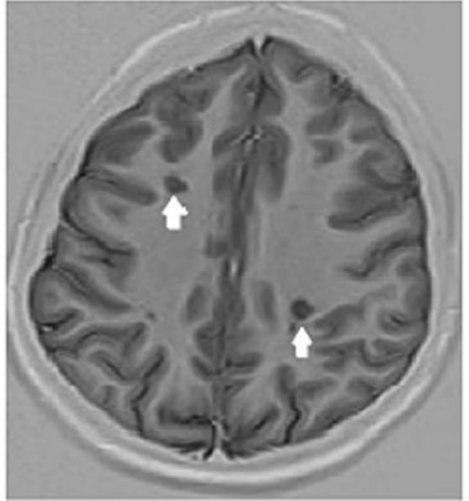

(c) $T I I R$

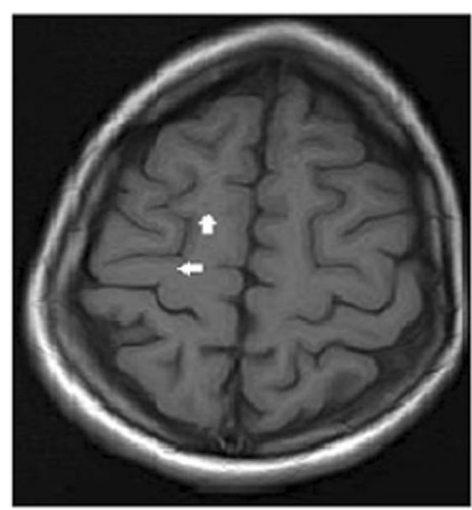

(b) TISE

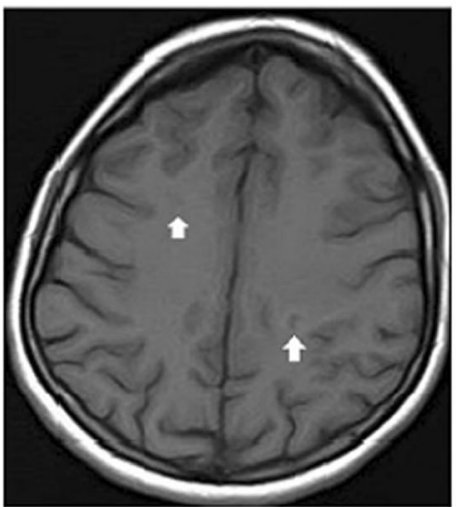

(d) TISE

Fig. 4 Known case of multiple sclerosis for follow-up. a T1IR. b T1SE. T1IR aided in the detection of juxtacortical black hole lesions (arrows in a) that were not detected in T1SE (arrows in b). c T1IR. d T1SE. Hypointense black hole lesions were better identified and delineated in T1IR (arrows in $\mathbf{c}$ ) than in T1SE (arrows in $\mathbf{d}$ )

values $1.29(0.16)$ vs $1.29(0.16)(p$ value $=0.5)$ and 2.19 (0.94) vs 1.75 (0.57) ( $p$ value 0.13 ; Table 4$)$.

\section{Discussion}

Conventional MR sequences produce only positive longitudinal magnetization. Conversely, inversion recovery

Table 2 Difference between T1IR and T1SE in CNR based on type of lesion

\begin{tabular}{llll}
\hline & MR sequences & Pre-contrast CNR & Post-contrast CNR \\
\hline Extra-axial lesion & \multicolumn{1}{c}{ T1IR } & $-13.90(2.86)^{*}$ & $13.17(3.01)$ \\
& T1SE & $-6.82(1.23)$ & $18.48(3.48)$ \\
& $p$ value & 0.02 & 0.06 \\
Intra-axial lesion & $\quad$ T1IR & $-12.87(2.40)^{*}$ & $16.46(4.07)$ \\
& T1SE & $-8.11(0.93)$ & $17.08(2.65)$ \\
MS lesion & $p$ value & $<0.05$ & 0.45 \\
& T1IR & $-12.69(1.63)^{*}$ & \\
& T1SE & $-8.25(1.35)$ & \\
& $p$ value & 0.01 & \\
\hline
\end{tabular}

${ }^{*} p$ value $<0.05$
(IR) sequences are displayed after rotating the positive longitudinal magnetization into negative magnetization, so IR sequences can generate more tissue contrast [2]. T1IR is an MRI sequence with 180 radio-frequent pulses with high lesion CNR and GM-WM contrast ratio than T1SE sequence, so it can be used in the evaluation of various neoplastic space-occupying lesions and demyelinating diseases such as multiple sclerosis [1]. In our study, we compared between T1IR and T1SE on 50

Table 3 Difference between T1IR and T1SE in CNR based on enhancement ratio(ER)

\begin{tabular}{lll}
\hline & MR sequences & Post-contrast CNR \\
\hline High enhancement & T1IR & $0.64(0.37)$ \\
& T1SE & $21.24(1.92)^{*}$ \\
$p$ value & & $<0.01$ \\
Low enhancement & T1IR & $18.91(2.68)^{*}$ \\
& T1SE & $1.05(0.59)$ \\
$p$ value & & $<0.01$
\end{tabular}

${ }^{*} p$ value $<0.05$ 
Table 4 Difference between T1IR and T1SE based on number of lesions

\begin{tabular}{lll}
\hline & MR sequences & Number of lesions \\
\hline Extra-axial lesion & T1IR & $1.29(0.16)$ \\
& T1SE & $1.29(0.16)$ \\
& $p$ value & 0.5 \\
Intra-axial lesion & T1IR & $2.19(0.94)$ \\
& T1SE & $1.75(0.57)$ \\
MS & $p$ value & 0.13 \\
& T1IR & $10.67(2.26)^{*}$ \\
& T1SE & $3.89(1.05)$ \\
& $p$ value & $<0.01$ \\
\hline
\end{tabular}

${ }^{*} p$ value $<0.05$

patients with different brain diseases aiming to reveal superiority of each sequence in each brain disease. In our study, on non-contrast sequences, T1IR was better than T1SE, giving higher CNR with mean value - 13.04 (1.20) for all types of lesions (multiple sclerosis, intra-axial, and extra-axial space-occupying lesions).

The same of our results were reported by Bing et al. [1] in a study carried on 30 patients with various neurologic diseases, and T1IR was superior to T1SE with improved determination of lesion extent, conspicuity, and lesion delineation; this was because T1SE had the poorest GM-WM contrast and poor lesion-to-background contrast by visual comparison between T1SE and T1IR. As well, Rydberg et al. [11], in a study carried on 15 patients with contrast-enhancing brain space-occupying lesions, showed that T1IR had quantitatively comparable or superior lesion-to-background contrast ratio, GM-WM contrast ratio, and superior CSF-WM contrast ratio; also, it provided qualitatively superior lesion detection compared to T1SE, as well as, superior lesion conspicuity and image contrast. In the same context, Lee et al. [12], in a study carried on 15 patients with 18 lesions, showed that T1IR was superior to T1SE in the determination of lesion extent in 16 lesions and provided higher CNR and GM-WM contrast ratio, but it takes more time than T1SE. However, Qian et al. [3] reported opposite results to our study, as T1SE was sensitive than T1IR in the detection of brain metastasis, but this can be accounted by the difference of nature of the disease, because many of metastatic lesions included in this study were small hemorrhagic lesions that were better detected on T1SE because of its sensitivity to hemorrhagic lesions.

We also reported in our study that after giving intravenous contrast media, T1IR was better than T1SE, giving higher CNR in lesions with low enhancement ratio with mean value 18.91 (2.68) vs 1.05 (0.59). On the other side, T1SE was better than T1SE in lesions with high enhancement ratio with mean value 21.24 (1.92) vs 0.64 (0.37). This goes on with results of Bandai et al. [13], in which T1IR provided higher tumor-to-WM contrast ratio with mean value 38.89 (2.68) in 13 enhanced tumors, and it also revealed 7 non-enhanced and low enhanced tumors that were poorly visualized on T1SE. The same results were reported in other studies $[14,15]$.

In our study, T1IR aided in the detection of higher number of lesions than T1SE especially in patients with multiple sclerosis with mean value 10.67 (2.26) vs 3.89 (1.05). This seemed consistent with Harel et al. [16], Sethi et al. [17], and Nelson et al [18] who showed that T1IR improved the detection of cortical and juxtacortical lesions in progressive multiple sclerosis with accurate localization of them; this can be due to the higher WMGM contrast ratio, making it extremely useful in followup of these patients.

We encountered some limitations in our study. Firstly, our study included only 50 patients; this number reveals minor differences between T1IR and T1SE especially with different brain lesions. Secondly, most of our MS patients were in inactive state. Therefore, we could not assess their SI on post-contrast lesions. Finally, we did not assess the size of lesions in both sequences. Hence, more studies should be done to reveal the full comparison between both sequences.

\section{Conclusion}

On pre-contrast sequences, T1IR could be used as an added sequence in most brain lesions giving higher lesion CNR. After giving intravenous contrast media, T1IR could be used in lesions with low ER. It also could be used in situations in which gadolinium injection is contraindicated and also could be used in the follow-up of MS patients by detecting a higher number of lesions that can be easily missed in T1SE.

\section{Abbreviations}

CNR: Contrast noise ratio; ER: Enhancement ratio; FLAIR: Fluid-attenuated inversion recovery; GM-WM: Gray matter-white matter; IR: Inversion recovery; MR: Magnetic resonance; MS: Multiple sclerosis; SI: Signal intensity; STIR: Short tau inversion recovery; T1IR: T1 inversion recovery; T1SE: T1 spin echo

\section{Acknowledgements \\ There is no acknowledgement.}

\section{Availability for data and materials}

The datasets used and analyzed during the current study are available from the corresponding author on reasonable request.

\section{Authors' contributions}

$\mathrm{MS}, \mathrm{RH}$, and $\mathrm{AE}$ contributed equally to the study design, data collection, analysis, and interpretation of results. All authors read and approved the final manuscript

Funding

This study had no funding from any resource. 


\section{Ethics approval and consent to participate}

All study procedures were conducted in accordance with the Declaration of Helsinki and were approved by the ethical committee of Menoufia Faculty of Medicine, and all data were extracted after taking written informed consent from patients involved in the study.

\section{Consent for publication}

All patients included in this research gave written informed consent to publish the data contained within this study.

\section{Competing interests}

The authors declare that they have no competing interests.

Received: 25 June 2019 Accepted: 30 July 2019

Published online: 17 September 2019

\section{References}

1. Hou P, Hasan KM, Sitton CW, Wolinsky JS, Narayana PA (2005) Phasesensitive $\mathrm{T1}$ inversion recovery imaging: a time-efficient interleaved technique for improved tissue contrast in neuroimaging. Am J Neuroradio 26(6):1432-1438

2. Sheppard S, Davis PC, Kater G, Peterson JE (1998) Inversion-recovery echoplanar MR in adult brain neoplasia. Am J Neuroradiol 19(2):267-273

3. Qian YF, Yu CL, Zhang C, Yu YQ (2008) MR T1-weighted inversion recovery imaging in detecting brain metastases: could it replace T1-weighted spinecho imaging? Am J Neuroradiol 29(4):701-704

4. Yousry TA, Filippi M, Becker C, Horsfield MA, Voltz R (1997) Comparison of MR pulse sequences in the detection of multiple sclerosis lesions. Am J Neuroradiol 18(5):959-963

5. Datta S, Sajja BR, He R, Wolinsky JS, Gupta RK, Narayana PA (2006) Segmentation and quantification of black holes in multiple sclerosis. Neuroimage 29(2):467-474

6. Healy BC, Buckle GJ, Ali EN, Egorova S, Khalid F, Tauhid S, Bakshi R (2017) Characterizing clinical and MRI dissociation in patients with multiple sclerosis. J Neuroimaging 27(5):481-485

7. Klawiter EC (2013) Current and new directions in MRI in multiple sclerosis. Continuum 19(4 Multiple Sclerosis):1058

8. Farshidfar Z, Faeghi F, Haghighatkhah HR, Abdolmohammadi J (2017) The optimization of magnetic resonance imaging pulse sequences in order to better detection of multiple sclerosis plaques. J Biomed Phys Eng 7(3):265

9. Simon B, Schmidt S, Lukas C, Gieseke J, Träber F, Knol DL, Wattjes MP (2010) Improved in vivo detection of cortical lesions in multiple sclerosis using double inversion recovery MR imaging at 3 Tesla. Eur Radiol 20(7):1675-1683

10. Filippi M, Baratti C, Yousry T, Horsfield MA, Mammi S, Becker C, Comi G (1996) Quantitative assessment of MRI lesion load in multiple sclerosis: a comparison of conventional spin-echo with fast fluidattenuated inversion recovery. Brain 119(4):1349-1355

11. Rydberg JN, Hammond CA, lii JH, Jack CR Jr, Grimm RC, Riederer SJ (1996) T1-weighted MR imaging of the brain using a fast inversion recovery pulse sequence. J Magn Reson Imaging 6(2):356-362

12. Lee JK, Choi HY, Lee SW, Baek SY, Kim HY (2000) Usefulness of T1-weighted image with fast inversion recovery technique in intracranial lesions: comparison with T1-weighted spin echo image. Clin Imaging 24(5):263-269

13. Bandai H, Tsunoda A, Mitsuoka H, Arai H, Sato K, Makita J (2002) Fast inversion recovery magnetic resonance imaging with the real reconstruction method: a diagnostic tool for cerebral gliomas. Neurol Med Chir 42(1):5-10

14. Fischbach F, Bruhn H, Pech M, Neumann F, Ricke J, Felix R, Hoffmann KT (2005) Efficacy of contrast medium use for neuroimaging at 3.0 T: utility of IR-FSE compared to other T1-weighted pulse sequences. J Comput Assist Tomogr 29(4):499-505

15. Batouli A, Kanal E, Gholamrezanezhad A, Spearman M (2018) T1-weighted parenchyma attenuated inversion recovery: a novel sequence that improves contrast ratio of enhancing brain lesions. Diagn Interv Imaging 99(1):29-35

16. Harel A, Ceccarelli A, Farrell C, Fabian M, Howard J, Riley C, Inglese M (2016) Phase-sensitive inversion-recovery MRI improves longitudinal cortical lesion detection in progressive MS. PloS one 11(3):e0152180

17. Sethi V, Yousry TA, Muhlert N, Ron M, Golay X, Wheeler-Kingshott C, Chard DT (2012) Improved detection of cortical MS lesions with phase-sensitive inversion recovery MRI. J Neurol Neurosurg Psychiatry jnnp-2012.
18. Nelson F, Poonawalla AH, Hou P, Huang F, Wolinsky JS, Narayana PA (2007) Improved identification of intracortical lesions in multiple sclerosis with phase-sensitive inversion recovery in combination with fast double inversion recovery MR imaging. Am J Neuroradiol 28(9):1645-1649

\section{Publisher's Note}

Springer Nature remains neutral with regard to jurisdictional claims in published maps and institutional affiliations.

\section{Submit your manuscript to a SpringerOpen ${ }^{\circ}$ journal and benefit from:}

- Convenient online submission

- Rigorous peer review

- Open access: articles freely available online

- High visibility within the field

- Retaining the copyright to your article

Submit your next manuscript at $\boldsymbol{\nabla}$ springeropen.com 